International Journal of Advanced Trends in Computer Science and Engineering, Vol.2, No.1, Pages : 576 - 582 (2013)

Special Issue of ICACSE 2013 - Held on 7-8 January, 2013 in Lords Institute of Engineering and Technology, Hyderabad

\title{
KNOWLEDGE MANAGEMENT STRATEGIES
}

\author{
Dr. G. Chandra Sekhar \\ Professor\& Head, \\ School of Management Studies, \\ Joginpally B.R. Engineering College, Hyderabad \\ Chandrasekhar.gotlagunta@gmail.com \\ Ms.S.Sujatha \\ Associate Professor \& Head \\ Department of Management studies \\ Lords Institute of Engineering \& Technology \\ Ph: 8688442722 \\ Sujatha.mbaliet@gmail.com
}

\begin{abstract}
$K M$ in practice seems to be an effort that comprises all kinds of different activities, measures and technologies. Unfortunately, it seems that organizations do not pay very much attention to the strategic value of their initiatives.

Thus, there is a lack of clear distinction between different KM strategies or dimensions of strategies that can be used to characterize one particular KM strategy.

This paper is made an attempt to describe about KM strategies and practice as well. This research paper discuses different types of repositories and role of repositories in $K M$, further it addresses incentives to make use of good repository records.
\end{abstract}

\section{KNOWLEDGE MANAGEMENT STRATEGIES}

In the learning literature, the nature of the market environment is a critical factor (e.g. Baldwin et al., 1997). In general, in low environmental turbulence conditions, the perceived need for change and the need for new information are likely to be low, and low-level learning (e.g. employee development) may be acceptable. Alternatively, under conditions of high environmental turbulence, the perceived need for change, for information, and for higher-order learning is likely to be higher. There appears to be a paradox. While

strategic alliances are implemented more efficiently when there is minimum tension between the partners, knowledge creation is maximized when such tension is increased (Phan an Peridis, 2000). Therefore, the greater the perceived turbulence in the business environment, the greater the need for inter-organizational learning.

Knowledge Management (KM) and Organizational Memory (OM) are concepts 
International Journal of Advanced Trends in Computer Science and Engineering, Vol.2, No.1, Pages : 576 - 582 (2013) Special Issue of ICACSE 2013 - Held on 7-8 January, 2013 in Lords Institute of Engineering and Technology, Hyderabad

well known from organization science and learning theory. Many approaches have been developed which claim to guide organizations to use their common or shared memory in a more efficient way. With the advent of advanced database technologies, net and communication technologies, especially the so-called "Intranet"- or "Web"-technologies, as well as dedicated knowledge management systems, sound information and communication technologies exist to support organizational processes of creating, acquiring, and organizing, distributing and applying knowledge. There are already a large number of KM activities implemented in organizations which often lack a strategic perspective. KM seems to "absorb" all kinds of theoretical approaches as well as practical activities, measures and technologies without very much deep consideration as to its strategic or business value. We hypothesize that an organization should

follow a complex KM strategy as part of a comprehensive business strategy. A KM strategy can be described using several dimensions derived from a theoretical and empirical survey of KM activities, measures and technologies. Process orientation is a perspective widely accepted in organization science. However, up to now there is no link between the two concepts on the strategic level. Organizations which have already implemented a process-oriented organizational design can use processorientation as one of the strategic dimensions.

KM strategies can be distinguished according to the type of knowledge that is focused. In order to position an organization against its competitors, the following three categories of knowledge can be distinguished per area of competency, or per strategic business unit, division,

product line, function or market position: core knowledge: the minimum knowledge commonly held by members of an industry; also considered the basic industry knowledge barrier to entry; advanced knowledge: enables an organization to be competitively viable; competitors may generally hold about the same level, scope or quality of knowledge, but knowledge differentiation can take place with competitors holding specific knowledge; innovative knowledge: enables an organization to lead its industry and to significantly differentiate itself from its competitors. Along these lines a large number of "dimensions" can be distinguished which describe various types of knowledge. These knowledge processes are transformations of knowledge of one type into knowledge of the opposite type of one and the same pair. A number of knowledge dimensions can be distinguished with respect to the corresponding main "area of intervention" organization, systems, content etc.

\section{Knowledge Management Strategies in Practice}

This section presents some empirical results concerning KM goals and strategies as well as the relationship to business strategy. The results were in a literature survey. The empirical study investigated the state-of-the-art of the use of KMS in the 500 largest German companies and the top 50 
International Journal of Advanced Trends in Computer Science and Engineering, Vol.2, No.1, Pages : 576 - 582 (2013) Special Issue of ICACSE 2013 - Held on 7-8 January, 2013 in Lords Institute of Engineering and Technology, Hyderabad

banking and insurance companies as well as the development of concepts, scenarios and reference models for the management of KMS in organizations. In 22 of the 73 responding organizations $(30,1 \%) \mathrm{KM}$ was well established.

With the exception of "improving innovation" which can be seen as a very general goal those KM goals that are focused strongly by most if not all organizations primarily try to

(a) improve the handling of existing knowledge in documents or in people's heads: "improve transparency" (17 organizations strongly aim at this goal), "improve access" (14), "improve documentation" (13) and "retention of knowledge" (14), or to

(b) improve the sharing of knowledge: "improve knowledge sharing" (12), "improve communication" (13). 12 out of 18 organizations $(66,7 \%)$ aimed at eight or more KM goals strongly at the same time. It seems that KM initiatives are currently very broadly and vaguely defined projects. The rates of achievement of most of the KM goals were ranked on average between 3,71 and 4,63 (on a 7-point scale with 7 being the highest score) showing a medium level of achievement. Thus, it seems that the KM efforts of the responding organizations on average still have some way to go until the more advanced benefits can be harvested. Concerning the relationship of KM initiatives to business goals the highest benefits are estimated to be in the rather "soft" areas like "improve customer satisfaction", "improve speed of innovation" whereas the "hard criteria" do not achieve equally high estimates (e.g. "reduce costs", "improve growth of organization"). It is interesting that two of the three highest ranked goals ("improve customer satisfaction", "improve productivity") are also typical business process reengineering goals.

It is not surprising that only 13 respondents (65\% of those responding to this question) did not respond on the question of business process, whereas 7 respondents indicated that they did not know how many business processes were targeted. In the remaining 53 cases the organizational design of the KM initiative was not (yet) detailed enough to cover this aspect. Of the 13 respondents 9 did not focus on business processes, but supported all business processes throughout the organization. The other 4 respondents focused 2, 3, 4 and 10 business processes ( 1 case per answer). As hypothesized it seems that process orientation is not yet focused in most of the KM activities of German organizations despite the fact that most organizations had already undergone process management programs in the past. There are also a number of authors who pragmatically suggest a series of KM activities, efforts or strategies without very much differentiation between these concepts. Most of these authors base their findings on empirical studies investigating $\mathrm{KM}$ initiatives in organizations. 
International Journal of Advanced Trends in Computer Science and Engineering, Vol.2, No.1, Pages : 576 - 582 (2013) Special Issue of ICACSE 2013 - Held on 7-8 January, 2013 in Lords Institute of Engineering and Technology, Hyderabad

- Map sources of internal expertise: the issue is to make knowledge assets visible, to increase managers' attention; the focus is on the personal side of the knowledge in an organization, e.g. expert directories, skill data bases, yellow pages.

- Establish new knowledge roles: create a separate organizational unit, create positions or roles responsible for knowledge-related tasks, such as knowledge engineer, assign personal responsibility for knowledge.

- Create a (virtual) work environment which enables the sharing of tacit knowledge: the issue is to create virtual workspaces, networks of knowledge workers which provide an alternative environment to the co-located workspace, thus enabling the sharing of tacit knowledge.

- Support knowledge flows in an organization: knowledge seekers and knowledge providers should be connected using systems and tools which provide for a balancing of pull and push of knowledge. KMS are needed which adapt to usage and communication patterns of knowledge seekers and providers.

- Knowledge management as a business strategy: KM is either integrated within the overall business strategy or treated as a separate business strategy in parallel with other strategies.

- Customer-focused knowledge: the aim of this strategy is to capture knowledge about customers, their needs, preferences, businesses, reactions to actions taken by the organization etc.
- Intellectual asset management strategy: the aim of this strategy is the enterpriselevel management of patents, technologies, operational and management practices, customer relations, organizational arrangements, and other structural knowledge assets.

- Innovation and knowledge creation: research and development is focused to enhance innovation and the creation of new knowledge. Put in a nutshell, the state-of-the-art of KM strategies in practice can be described as follows: there are already a large number of initiatives in organizations under way. They combine very different approaches and singular activities which are supposed to deliver business value by improving the way an organization handles knowledge.

$\mathrm{KM}$ in practice seems to be an effort that comprises all kinds of different activities, measures and technologies. Unfortunately, it seems that organizations do not pay very much attention to the strategic value of their initiatives.

Thus, there is a lack of clear distinction between different KM strategies or dimensions of strategies that can be used to characterize one particular KM strategy.

\section{Types of Repositories}

Many kinds of repositories are involved in knowledge reuse. Perhaps the most basic distinction is that between repositories of documents and repositories of data. Blair observed that retrieving information in the form of textual documents is fundamentally different than 
International Journal of Advanced Trends in Computer Science and Engineering, Vol.2, No.1, Pages : 576 - 582 (2013) Special Issue of ICACSE 2013 - Held on 7-8 January, 2013 in Lords Institute of Engineering and Technology, Hyderabad

retrieving data. Consequently, strategies for indexing and storing the different kinds of information must differ as well. Increasingly, this basic distinction must be augmented with graphical information such as engineering drawings and with audio, video, and multimedia "documents."

Davenport and colleagues distinguish among repositories that store external knowledge (e.g., demographic data, competitive intelligence), those that store structured internal knowledge (in the form of data or documents), and those that store informal information (such as transcripts of group discussions via electronic meeting systems, computer conferencing, or electronic mail). Alavi and Leidner mention several specific kinds of (semi) structured internal knowledge repositories, including corporate yellow pages and people information archives.

A further way of classifying repositories is by means of the kind of knowledge they contain. General knowledge (including, for example, explicit scientific knowledge) and specific knowledge (including knowledge of the local context) may differ as is the case between technical and contextual knowledge.) Moorman and Miner distinguish between declarative knowledge (knowledge about facts) and procedural knowledge (knowledge about how things are done). To this distinction must be added rationale knowledgeknowledge about why things were done, as in the design of software products and analytic knowledge-the conclusions reached by applying declarative and procedural knowledge to a particular fact domain.

\section{The Role of Repositories in Knowledge Management}

Different types of knowledge reusers need different things from knowledge repositories. Shared work producers and shared work practitioners need contextualized knowledge (although the former can more effectively use raw records than the latter, who require more "sanitized" and quality-checked records). Expertiseseeking novices needed decontextualized knowledge, knowledge about what contextual information is useful, and help recontextualizing the information for their unique settings. Secondary knowledge miners can benefit from, but usually have to make do without, in-depth knowledge of the contextual influences on the creation of records stored in repositories. However, the different types of knowledge reusers do not always get what they need from repositories, for reasons that have to do in part with how repositories are created. Contents of repositories tend to differ when knowledge producers knowingly create records for different types of users. (In some cases, these differences may help the consumers. In other cases, a great deal of effort is required to produce repositories that meet users' needs, and that knowledge producers, who are frequently expected to produce high quality repositories, often lack both the motivation and the resources to do so. Therefore, successful knowledge reuse requires providing proper incentives for the knowledge producers and shifting some of the burden of packaging and disseminating 
International Journal of Advanced Trends in Computer Science and Engineering, Vol.2, No.1, Pages : 576 - 582 (2013) Special Issue of ICACSE 2013 - Held on 7-8 January, 2013 in Lords Institute of Engineering and Technology, Hyderabad

knowledge onto intermediaries. The purpose and content of records in repositories often differ depending on whether the record keepers are knowingly documenting only for themselves, for others who are similar to themselves in work product or practice community

\section{Incentives to Make and Use Good Repository Records}

The costs involved in creating and using repositories would not necessarily be a problem if they were balanced by appropriate incentives. But in many cases they are not. Grudin made this point generally for systems that support collaborative work, including shared work product and shared work practice situations. Ackerman particularizes Grudin's observation for the organizational memory systems that are the focus of this paper. Ackerman says organizational memory systems are subject to the issue of incentives. Grudin has pointed out the complexities of upstream versus downstream costs in adopting and using group and organizational systems. To the extent that OMS require upstream costs, such as those in indexing, and to the extent that the downstream payoffs are unclear, they will likely fail. The use of organizational memory adds to the cost. Not only is there the cost of storage and indexing, there may be additional costs in retrieval and interpretation of the information. The situation in which people contribute to a knowledge repository that both they and others can use has been described as the "discretionary database problem". The discretionary database problem has frequently been observed in the shared work practitioner situation such as the use of knowledge repositories implemented in consulting firms. The challenge is to ensure that people make high quality contributions without free riding on the efforts of others, particularly when people are pressed for time or competing with each other on the basis of performance. Orlikowski, for example, found that consultants in the hectic, up-or-out environment of the firm named Alpha did not use Lotus Notes as extensively as expected for knowledge sharing. Lack of appropriate incentives to contribute was implicated in the failure. Conversely, providing appropriate incentives has often been proposed as a solution to the discretionary database problem. In many consulting firms, explicit attention to extrinsic rewards has been found necessary to promote contributions to shared knowledge repositories. At Booz-Allen: "Consultants contribute for many reasons. But two reasons stand out: the system enhances their work and enhances their reputation among their colleagues". But both of these motivations are reinforced by the explicit reward systems in the firm: The job of persuasion is made easier by the firm's reward structure. Some practices offer awards for excellent contributions. These range from tombstones for the consultant's desk to the CMT practice's award of $\$ 50,000$ to a winning team for them to spend on learning-related activities of their own choice. The only restriction is that they must report back their learning to the practice as a whole. But more significantly, developing intellectual capital has become one of the 
International Journal of Advanced Trends in Computer Science and Engineering, Vol.2, No.1, Pages : 576 - 582 (2013) Special Issue of ICACSE 2013 - Held on 7-8 January, 2013 in Lords Institute of Engineering and Technology, Hyderabad

four criteria used when determining promotion and bonuses. Nevertheless, however useful they are, explicit rewards may be insufficient in the face of unsupportive organizational norms:

\section{Conclusion:}

India is now placed uniquely, with rest of the world keenly observing whether it can jump strait to become "service economy from agriculture economy", without much efforts on manufacturing sector it will be possible if our business leaders can mesh KM dimension with there business strategies. Collaborative strategies to develop KM strategies can go a long way. Strategic alliances represent looser and therefore less obvious learning entities than individual organizations, but this does not mean that organizations cannot learn collectively Interorganizational (joint) learning can be viewed as the collective acquisition of knowledge among a set of organizations: symbiotically interdependent, yet semiautonomous organizations that interact to construct or modify their collective environment, working rules, and options. If India can become back office to the world supporting networks, databases, repositories etc., should it not use that expertise to benefit from KM? We believe in a strong 'YES'.

\section{REFERENCES}

1. Alessandra Molani, Annaperini, Eric $\mathrm{Yu}$ and Paolo Bresciani "Analyzing the
Requirements for Knowledge Management using Intentional Analysis"

2. Babita Gupta, Lakshmi S. Iyer and Jay E. Aron son (2000) "Knowledge Management: Practices and Challenges". Industrial Management and Data Systems, pp. 17-21.

3. Eli Jones, Lawrence B, Chonko and James A. Roberts 2003, "Creating a Partnership- Oriented, Knowledge Creation Culture in Strategic Sales Alliances: a Conceptual Frame Work" Journal of Business and Industrial Marketing, Vol. 18, No. 4/5, pp. 336352.

4. Mary Johatch and Majkeen Schultz "Relations between Organizational Culture, Identity and Image" Europen Journal of Marketing, pp. 356-364.

5. Matteo Binifacio and Paolo Bouquet and Roberta Cuel "Knowledge Nodes : The Building Blocks of a Distributed Approach to Knowledge Management"

6. M. Lynne Markus (2001) "Toward a Theory of Knowledge Reuse : Type of Knowledge Reuse Situations and Factors in Reuse Success". Journal of Management Information Systems, Vol. 18, No. 1, pp. 57-93.

7. Ronald Maier, Ulrich Remus 2001 "Towards a Frame Work for Knowledge Management Strategies : Process Orientation as Strategic Starting Point". Proceedings of IEEE. pp. 1-10. 